TITLE:

\title{
Mutant cells defective in DNA repair pathways provide a sensitive high- throughput assay for genotoxicity.
}

\section{$\operatorname{AUTHOR}(S)$ :}

Evans, Terry John; Yamamoto, Kimiyo N; Hirota, Kouji; Takeda, Shunichi

\section{CITATION:}

Evans, Terry John ...[et al]. Mutant cells defective in DNA repair pathways provide a sensitive high-throughput assay for genotoxicity.. DNA repair 2010, 9(12): 1292-1298

\section{ISSUE DATE:}

2010-12-10

URL:

http://hdl.handle.net/2433/134555

\section{RIGHT:}

(C) 2010 Elsevier B.V.; This is not the published version. Please cite only the published version.; この論文は出版社版でありません。引用の際に は出版社版をご確認ご利用ください。 
Mutant cells defective in DNA repair pathways provide a sensitive high-throughput assay for genotoxicity.

Terry John Evans, Kimiyo Yamamoto, Kouji Hirota and Shunichi Takeda*

Department of Radiation Genetics, Graduate School of Medicine, Kyoto University, Yoshidakonoe, Sakyo-ku, Kyoto 606-8501, Japan

*Correspondence: E-mail: stakeda@rg.med.kyoto-u.ac.jp phone: +81-75-7534410; fax: +81-75-753-4419. 


\section{$\underline{\text { Abstract }}$}

Chemicals used industrially and commercially are required by law to be assessed for their genotoxic potential. However, all currently used assays have major limitations and despite intense effort, there is no universal agreement on which tests should be employed, or how to interpret results. We have developed a new assay system using the chicken DT40 B cell line that offers a number of significant advantages over current methodologies. Our assay could provide enhanced sensitivity by using genetically defined and phenotypically characterized mutants defective in DNA repair pathways. Furthermore, analysis of the mutants, using DNA repair proficient wild-type cells as a negative control, minimizes false negative outcomes. Assessing the different responses of a panel of mutants representative of all repair pathways, mechanistic detail of genotoxicity can be determined. This unique feature, as well as reducing the false positive rate, strengthens positive identifications and is useful when extrapolating results to the human context. Our panel of mutants is likely to be useful in screening large compound libraries for an emerging class of chemotherapeutic drugs, which includes inhibitors of DNA repair enzymes such as PARP and DNA polymerases.

\section{$\underline{\text { Introduction }}$}

We have used the chicken DT40 B lymphocyte cell line to investigate the mechanisms of the DNA damage response, both resulting from endogenous damage and in response to chemotherapeutic (genotoxic) agents. Through our reverse genetic studies - we have analyzed mutants deficient in all the DNA repair pathways - many aspects of the cellular DNA damage response have been elucidated. Most DNA repair-deficient mutants are hypersensitive to DNA damaging agents, which led us to realize that these strains could be used as an assay system to identify putative genotoxins with greater sensitivity than existing assays. The strength of our system is that a diverse range of repair mutants has been challenged with many toxins, and the phenotypic consequences have been characterized in detail. In this article, we present our bioassay, which is both quantitative and high-throughput, and also provides the genotoxic mechanism of action. In addition, we describe an important application of this system in the screening of chemical libraries for novel cancer therapeutics.

\section{DNA damage response pathways associated with DNA replication}

Mutagenic chemical compounds cause mutations and chromosomal aberrations during DNA replication through the following mechanisms. The vast majority of DNA damage stalls replicative DNA polymerases, and occasionally causes the collapse of DNA replication, leading to chromosomal breaks in mitotic cells and subsequent cell death. To release stalled DNA synthesis, several TLS DNA polymerases undergo a few nucleotides DNA synthesis over damaged template strands, followed by re-initiation of DNA synthesis by replicative DNA 
polymerases. Thus, stalled replication results in the accumulation of point mutations through the action of error-prone translesion DNA polymerases. Homologous recombination also contributes to the re-initiation of DNA synthesis. In additional to HR and TLS, reinitiation of DNA synthesis at replication blockages caused by interstrand crosslinks requires the Fanconi anemia repair pathway. Thus, mutagenic chemical compounds cause the collapse of DNA replication to considerably higher extent in cells deficient in the translesion DNA synthesis, HR, and FA pathways in comparison with wild-type cells. Accordingly, following exposure of cells to mutagenic chemical compounds, cells deficient in the translesion DNA synthesis, homologous recombination, and Fanconi anemia pathways display significantly greater numbers of chromosomal aberrations than do wild-type cells.

In addition to the repair pathways associated with DNA replication, there are a number of DNA repair pathways that are functional throughout the cell cycle. These include base excision repair, nucleotide excision repair, and nonhomologous end joining. The panel of DNA repair mutants used in a published study [1] included DT40 mutants in the following repair pathways: DNA damage checkpoint (ATM), homologous recombination (Rad54, XRCC3, and UBC13), non-homologous end joining (Ku70), interstrand cross-link repair (FANCC), translesion synthesis (Rev3), nucleotide excision repair (XPA and XPG), and base excision repair (DNA Polymerase $\beta$ ). Since individual repair pathways process distinct sets of the DNA lesion type, the panel of DT40 mutants would contribute to the characterization of DNA lesions induced by chemical compounds as well as to the detection of mutagenic chemical compounds.

\section{The chicken DT40 cell line for investigating DNA repair}

The DT40 B lymphocyte cell line has proved to be a very tractable experimental system. Genetic deletions are comparatively easy to obtain owing to the high efficiency of targetted integration [2,3]. Unlike some other cell lines, DT40 displays a remarkably stable karyotype (and phenotype), even during prolonged passage. Conveniently, DT40 cells display a short doubling time ( $\sim 8$ hours) and grow in suspension.

A number of features of DT40 cells are particularly relevant to our research into DNA repair and their use in genotoxicity testing. First, DT40 cells have an unusually long S phase - about $70 \%$ of the cell cycle. This means that the majority of cells treated with DNA damaging agents will be in the process of synthesising DNA. Second, the lack of a functional p53 means that the G1/S checkpoint is not activated and DNA damage accumulates as the cell cycle progresses without complete repair (albeit at a reduced rate). In contrast, longer G1 phases in other cell types permit DNA repair before entry to $S$ phase without extending the cell cycle. It is important to appreciate that even DNA damage that occurs in G1 phase usually interferes with replication in DT40 cells.

\section{Current genotoxicity tests and their limitations}


No single genotoxicity test satisfies the regulatory bodies of every country. Indeed, disagreement has been publically expressed over the testing guidelines recommended by the European Scientific Committee on Cosmetics and NonFood Products, for example [4]. Ethical and institutional demands to reduce the number of animals used in research has resulted in initiatives such as the European Cosmetics Directive, requiring that some in vitro studies are not followed up in vivo. Regulatory decisions made solely on evidence from in vitro studies therefore hinge on their reliability. However, current in vitro tests suffer from many limitations; in particular, the rate of both false positive and false negative results remains unacceptably high, as exemplified by the screening of mutagenic chemical compounds described in the following paragraphs.

Whilst vigorous debates regarding genotoxicology continue in such forums as the International Workshops for Genotoxicity Testing and the International Conference on Harmonization of Technical Requirements for the Registration of Pharmaceuticals for Human Use [5,6], the only consensus that has been reached is the need for further improvement on current methods. Guidance on which methods to use and how to interpret the data is regularly updated, and as the science advances, so the recommended list of genotoxicity assays is altered to better identify chemical compounds of concern [eg 7].

In a thorough survey, Kirkland et al. have reviewed the genotoxicity of over 700 different chemicals that had been assayed using a variety of tests [8], focussing on the most commonly used assays:

- the Ames test (a bacterial reverse mutation assay);

- the mouse lymphoma assay (a negative selection for loss of the functional thymidine kinase gene); and

- either the in vitro micronucleus assay (detection of additional small nuclei) or chromosomal aberration assay.

Kirkland et al. assessed the performance of these tests, and this enabled the rate of false positives and false negatives to be compared; we have summarized their findings in Table 1 . It is clear that no single test was sufficiently sensitive to detect all known rodent carcinogens, and specificity levels were also worryingly low. A major conclusion of their work is that the use of more than three tests is discouraged because, whilst the sensitivity of such a group of tests increases, the number of false positive results also rises dramatically.

The Ames test is the simplest and quickest of the existing genotoxicity assays. In essence, it is a bacterial reverse mutation assay that is primarily capable of detecting point mutations and frame shift mutations, but notably not chromosomal rearrangements or double strand breaks. The Ames test came under attack nearly as soon as it was described for the first time, and discussions in the literature regarding its suitability have been lively, mostly on the basis that mutagenicity does not necessarily correlate with carcinogenicity [9]. Despite its widespread adoption, numerous reports describe how this bacterial test is not a suitable model for the eukaryotic system. Nonetheless, various modifications to the Ames test, such as the use of liver homogenate for the metabolic (de)activation of potential genotoxins have contributed to its success $[10,11,12]$, but its convenience is probably the overriding factor in its popularity. 
The micronucleus assay is particularly prone to false positive and false negative results. In this assay, double strand breaks result in chromosomal fragments that are not attached to microtubules during metaphase, and are therefore not pulled to opposite poles before cell division. These so-called lagging chromosome fragments migrate outside the normal nucleus and can be observed microscopically as structures known as micronuclei. False negative results are caused by the fact that the micronucleus assay detects only double strand breaks. Accordingly, although UV irradiation is highly mutagenic, UV photoproducts do not directly generate micronuclei, unless UV photoproducts cause replication collapse leading to chromosomal breaks. False positive results occur when an undamaged but lagging chromosome also forms a micronucleus. For example, taxol (which is a cancer chemotherapy) inhibits the depolymerization of microtubules. This interferes with their dynamic instability, which is essential for the correct partitioning of sister chromatids after replication. Similarly, colcemid inhibits microtubule polymerization, and is also detected as a genotoxin by this assay. To exclude the false-positive results, we here propose the use of DNA repair-deficient cells in the micronucleus assay using repairproficient cells as a negative control. The employment of DNA repair-deficient cells would also increase the sensitivity of the micronucleus assay, as discussed in the next paragraph.

\section{The logic of the DT40 genotoxicity assay}

By using the DT40 system, chemicals that directly cause DNA damage, and thus micronuclei, can be distinguished from compounds such as taxol by comparing the number of micronuclei in wild type DT40 and DNA repair-deficient cells (Figure 1). Taxol should cause micronucleus formation in wild type cells, just as it currently does in the mouse-derived cells used in this assay. However, the number of micronuclei should not be significantly increased in repair-deficient DT40 mutants because repair proficiency is not related to micronucleus formation in this case. In contrast, true genotoxins would cause more micronuclei in repair-deficient backgrounds. The DT40 system can also be used to ascertain if spindle defects are the cause of micronuclei by analyzing mutants partially deficient in the kinetochore. In this case, chemicals causing one or a few micronuclei in the wild type cells would cause multiple micronuclei in kinetochore mutants.

Using DNA repair deficient cells allows for the increased sensitivity of the micronucleus assay. In the cells used in the micronucleus assay, UV damage triggers G1/S arrest, which prevents DNA replication unless the majority of the induced DNA lesions are repaired. Thus, an efficient G1/S checkpoint may significantly reduce the sensitivity of the micronucleus assay to detect environmental mutagens. Since the G1/S checkpoint does not exist in DT40 cells, replication and cell division occur even in the presence of DNA damage, and micronuclei can be detected reliably.

Conventional genotoxicity analyses, such as the micronucleus assay, require that the method can selectively detect DNA damage and exclude other types of chemical toxicity. On the other hand, to analyze cellular responses to well- 
characterized known DNA damaging agents, such as X-rays and UV, researchers firstly measure cellular survival following exposure of cells to these damaging agents. However, this survival assay cannot be used for screening mutagenic compounds because it is not specific for detecting genotoxicity. This problem would be solved if cellular survival is compared between DNA repair proficient wild type cells and DNA repair mutants following exposure of cells to chemical compounds. The most promising mutant cells may be cells deficient in translesion DNA synthesis (TLS), because the vast majority of DNA lesions inhibit DNA synthesis by replicative DNA polymerases, and the efficient release from this inhibition is dependent on TLS DNA polymerases. Moreover, the hypersensitivity of TLS mutant cells to given chemicals indicate an important role of TLS polymerases in the tolerance of wild type cells to these chemicals. Since TLS polymerases are extremely error-prone and responsible for the majority of spontaneously arising mutations in the budding yeast, this hypersensitivity also indicates that these chemicals cause the accumulation of mutations through the action of TLS polymerases [13].

To exemplify the point discussed in the previous paragraph, we were able to detect the genotoxicity of therapeutic concentrations of tamoxifen, a widely used treatment for breast cancer. Tamoxifen acts by antagonizing the proliferative effects of oestrogen on oestrogen receptor-positive tumours. Recent guidelines recommended its use for 5 years following the surgical removal of tumours, and it is also prescribed prophylactically to women with a high risk of developing breast cancer. Worryingly, the incidence of secondary cancers, particularly endometrial cancer, is significantly higher in women following long-term exposure to tamoxifen, indicating a possible genotoxic side effect [14]. However, studies have variously reported both the presence and absence of DNA adducts in response to tamoxifen [14]. We addressed this unresolved issue by comparing the effects of this drug on DT40 clones deficient in various DNA repair pathways [15]. This demonstrated that defects in translesion synthesis (particularly a mutation in rev3, the catalytic subunit of the error-prone TLS polymerase Pol $\zeta$ ) resulted in hypersensitivity to tamoxifen. This manifested as an increased number of chromosome breaks, which is known to occur when lesions are not repaired post-replicatively at a stalled replication fork. Wild type cells, however, are not deficient in rev3. These data therefore suggest that TLS contributes to the prevention of chromosome breaks caused by tamoxifen. Because Pol $\zeta$ is particularly error-prone $[13,16]$, tamoxifen is tolerated in the short term, but this likely comes at the expense of mutations and chromosomal instability in the long term [17].

\section{Mechanism of action of putative genotoxins}

Information regarding the mode of action of mutagens and drugs helps satisfy regulatory bodies when making decisions based solely on in vitro assays; this is increasingly important as less reliance is put on animal testing [18]. In this regard, the simple, robust and informative phenotypic assays performed on DT40 DNA repair mutants are a prominent feature of the follow-up procedures possible with this system [19]. Indeed, extreme caution must be exercised when 
making inferences about mechanisms of action based on unestablished experimental procedures. One such "mechanism" for treating genetic disorders caused by nonsense mutations [20] was later disproved as an artefact [21].

Our assay exploits the fact that different cellular pathways are used to repair different types of DNA lesions. In a mutant cell line that is unable to repair a specific type of lesion, cells are markedly more sensitive to the causative genotoxic agent, resulting in reduced proliferation of a culture. The wild-type and mutant responses can be compared and this comparison serves four purposes: the wild-type is minimally sensitive to the chemical and provides a negative control; the mutant provides sensitive detection of the genotoxin; the comparison between wild type and mutant response permits the use of various high throughput methods, such as measurement of cellular survival, to detect the genotoxicity of chemicals (discussed below); and the mutant identity of the hypersensitive strain identifies the repair pathway responsible for damage repair, and thus the type of lesion produced.

Some genotoxins act via more than one mechanism, as was found to be the case for sodium metaarsenite. Arsenic contamination of drinking water is a significant problem across the world, predominantly in developing countries, but many locations in the USA also have water contaminated with arsenic at levels higher than those prescribed by the Environmental Protection Agency [1]. Consequently, knowledge of its toxic mechanisms is important because although arsenic is a known carcinogen, it is not detected in the Ames test [22]. Using DT40 cells, we showed that arsenic causes double strand breaks as well as lesions repaired by the same pathway that repairs UV-induced damage (nucleotide excision repair; NER). It was possible that the UV-like lesions were subsequenty converted to double strand breaks at a stalled replication fork. However, we disproved this possibility because arsenite hypersensitivity was ameliorated by the addition of the antioxidant $\mathrm{N}$-acetyl cystein specifically in DSB repair mutants was ameliorated, although this treatment had no effect on NER mutants (Figure 2). This example shows that a panel of DNA repair mutants are useful for dissecting complex mechanisms of mutagenesis by chemical compounds.

\section{High-throughput format of the assay}

Even the relatively rapid eukaryotic tests are labour-intensive. This limits the number of compounds as well as the range of chemical concentrations that can be assayed. Both of these issues are important considerations. Firstly, if a large number of chemicals could be screened for toxicity before initiating drug development, those lead molecules that are likely to fail toxicology screening could be eliminated at the outset. Secondly, because not all toxins are equally potent, they are usually screened at a high concentration $(10 \mathrm{mM}$ or 5000 $\mu \mathrm{g} / \mathrm{mL}$, according to Organisation for Economic Co-operation and Development guidelines) at which any physiologically relevant activity should be evident. However, there is disagreement over what the maximum concentration should be. Furthermore, high concentrations can lead to off-target effects that are not 
biologically meaningful. Such high drug concentrations are probably inappropriate for most compounds and should be revised downwards in order to reduce the number of false positive results, and thus increase the specificity of in vitro assays [23].

Our initial trial of this assay in HTP format was conducted in collaboration with the National Toxicology Program (NTP) in 1536-well plates in a total reaction volume of just $5 \mu \mathrm{L}$, and is automated. Because it is a high-throughput method, the drug concentration can be titrated over five orders of magnitude in the socalled quantitative high-throughput assay [24]. Bona fide toxins are expected to return classical dose-response curves and deviations from this pattern are easily detected, circumventing the problems associated with single-concentration toxicity screening. Indeed, Inglese et al. used the quantitative HTP assay to assess the proportion of false positives and false negatives that would be obtained from a single-concentration HTP screen [24]. For example, compounds that gave a negative result at $11 \mu \mathrm{M}$ (single-concentration HTP screens typically employ a drug concentration of $10 \mu \mathrm{M}$ [25]) but displayed a sigmoidal doseresponse curve at higher concentrations were deemed false negatives. In this manner, the false positive and false negative rates were found to be $2 \%$ and $40 \%$, respectively. Additionally, the dose-response data in genotoxicity investigations is important in risk assessment considerations, which take into consideration likely exposure levels [26].

The endpoint of this assay is production of light by luciferase, which reports on cellular ATP levels [27]. As ATP levels have been shown to correlate excellently with cell number over five orders of magnitude, it is a convenient proxy for this parameter and is associated with a high signal-to-noise ratio [27]. A thorough assessment of this luciferase-based assay in 1536-well format has shown it to be robust and highly reproducible over time [24].

More importantly, we have shown that direct counting of DT40 cells rather than colonies is able to detect some genotoxins at significantly lower drug concentrations and following a shorter incubation period - the $\mathrm{LD}_{10}$ for arsenite determined by colony counting was $1,400 \mu \mathrm{M}$ but only $45 \mu \mathrm{M}$ when assessed by direct cell counting at 48 to 72 hours after addition of chemical compounds [1]. Since the colony formation assay is the most reliable assay for DNA damage currently available, cell counting (indirectly, by quantifying ATP levels) represents a significant advance for the detection of genotoxicity using rapidly proliferating DT40 cells.

\section{$\underline{\text { Screening for new chemotherapeutic drugs }}$}

Currently, cancers are mostly treated by directly damaging DNA (for example, with X-rays or drugs such as cisplatin), which leads to cell death. This somewhat indiscriminate method also damages the DNA of healthy cells and often causes aggressive side effects. The specific targeting of cancer cells can be quantified with the therapeutic index, which is defined as the dose required for toxic effects divided by the dose required for therapeutic effects. A new class of chemotherapeutic drugs has begun to emerge which promises to be more 
successful in specifically targeting cancerous cells (ie, drugs with a high therapeutic index). These drugs, which can be used in combination with traditional agents that directly damage the DNA, target DNA damage repair pathways. In some cancers, these repair pathways are de facto defective (eg. familial breast and ovarian cancers are associated with defective homologous recombination due to mutations in BRCA1 and BRCA2 genes) or become compromised owing to the genomic instability and mutagenicity characteristic of most cancers. This makes cancer cells very sensitive to inhibition of the remaining repair pathways.

If two repair pathways are functionally redundant, loss of one pathway in tumours renders them entirely dependent on the remaining repair pathway. Chemical inhibition of the remaining pathway is expected to be toxic to the cancer cells but not to somatic cells. Using a combination therapy of DNA damaging agent and repair inhibitor is already proving successful, and is entirely analogous to the treatment of bacterial infections with amoxicillin (a $\beta$-lactam antibiotic) and clavulanic acid (an inhibitor of $\beta$-lactam breakdown). Inhibitors of specific repair pathways could be identified in this manner. For example, treatment of HR-deficient DT40 cells with cisplatin causes a dramatic decrease in cellular proliferation that is not worsened by simultaneous treatment with an HR inhibitor. However, since cisplatin conjugates can also be repaired via TLS, simultaneous treatment with cisplatin and a chemical inhibitor of TLS is predicted to have a very dramatic effect on cellular viability (Figure 3).

The inhibition of PARP-1 is an impressive example of this phenomenon. PARP-1 is involved in the repair of single strand breaks. If these are left unrepaired, they are converted to double strand breaks, and these require homologous recombination for their repair. Since as many as 40 different proteins are involved in HR, many cancers exhibit HR defects. Strikingly, it has been shown that inhibition of PARP-1 in a BRCA2-null background is lethal, even without exogenous DNA damage $[28,29]$. A number of PARP inhibitors are now in clinical trials.

This rationale has also been adopted for a variety of other cancer types. For example, germline mutations in the mismatch repair (MMR) genes MLH1 or MSH2 predispose to hereditary nonpolyposis colorectal cancer (also known as Lynch syndrome), which accounts for approximately $5 \%$ of all colorectal cancer cases; additionally, silencing methylation of the MLH1 promoter also appears to cause some colorectal cancers (reviewed by Jacob 2002 [30]). Lesions repaired by MMR can also be repaired by BER - a process that requires specialized DNA polymerases. In human cells, the DNA polymerases POLB and POLG are involved in the gap filling synthesis following 8-oxoguanine excision in the chromosomal and mitochondrial DNAs, respectively [31]. Inhibition of these polymerases in cells derived from MMR-deficient cancers, but not in non-cancerous controls, resulted in a synthetic lethal phenotype and therefore demonstrates the targetted killing of cancer cells, at least in this cancer type. Supporting these findings that polymerases play an important role in DNA repair, it has been shown that inhibition of DNA polymerases results in hypersensitivity of irradiated cells, and provides a further means of specifically sensitizing DNA repair-deficient cells to DNA damaging agents [32]. Furthermore, aberrant 
expression of mutagenic TLS polymerases was linked to a poorer prognosis for some cancer patients [33]. Unfortunately there are as yet no chemical inhibitors of these polymerases that are both potent and specific. Therefore, the DT40 assay could be useful in screening libraries for compounds with this effect.

Taken together, these advances in our understanding of cancer biology have lead to the suggestion that cancers should be considered from a repair-deficient viewpoint [34] in order to make more rational use of cancer therapeutics, rather than characterizing them based on their location, for example. This paradigm shift is easily modelled in DT40 cells because repair-deficient mutant strains are already available, and are amenable to experimental investigation.

\section{Future perspectives}

Whilst the DT40 assay is able to detect all types of genotoxicity, a single genotoxicity assay is unlikely to provide a complete genotoxic assessment. In particular, in vivo assays will remain important. Nonetheless, we feel that our system offers unique advantages and will find additional applications in toxicology. For example, the use of DT40 mutants displaying enhanced sensitivity to mitochondria or endoplasmic reticulum stressors could be used in the same high-throughput assay that has already been successfully applied to DNA repair mutants. Whilst not explicitly demonstrated in our system thus far, the use of specific repair-deficient mutants has elsewhere been shown to result in increased levels of drug-induced non-disjunction, underscoring the utility of this genetic approach to aneugens and clastogens [35].

The ultimate goal of detecting toxicity associated with test chemical compounds is to develop a method for in silico prediction of toxicity from their chemical structures. This in silico prediction depends on the high quality of the database. To obtain such a database, high-throughput screening is extremely useful. For example, comparing cellular survival of isogenic mutants in the presence of test chemicals yields reliable data that clearly indicate the functional relationship between disabled biochemical pathways in individual mutants and test chemical compounds. The value of such a database will surely increase with time.

In the spirit of continued improvement, we accept that the performance of our assay may be enhanced following further research. For example, the bioassay's sensitivity may be improved by creating a cell line deficient in more than one DNA repair pathway (providing that such cells were viable); alternatively, more sensitive molecular markers may be discovered that respond more dramatically to damage in the cell. In the meantime, our genetic method for detecting genotoxins whilst simultaneously clarifying the mechanism of toxicity is likely to have broad appeal. 
Table 1 . The specificity and sensitivity of current genotoxicity assays.

\begin{tabular}{|c|c|c|c|c|c|}
\hline & & & & & \\
\hline & Ames $^{1}$ & $\mathrm{MN}$ & MLA & $\begin{array}{l}\text { Ames \& } \\
\text { MN }\end{array}$ & $\begin{array}{l}\text { Ames, MN } \\
\text { \& MLA }\end{array}$ \\
\hline $\begin{array}{l}\text { Number of carcinogens } \\
\text { tested }\end{array}$ & 541 & 89 & 245 & 372 & 54 \\
\hline Sensitivity $(\%)^{2}$ & 58.8 & 78.7 & 73.1 & 94.4 & 90.7 \\
\hline False negative rate $(\%)$ & 41.2 & 21.3 & 26.9 & 5.6 & 9.3 \\
\hline $\begin{array}{l}\text { Number of non-carcinogens } \\
\text { tested }\end{array}$ & 176 & 26 & 105 & 15 & 20 \\
\hline Specificity $(\%)^{3}$ & 73.9 & 30.8 & 39.0 & 53.3 & 5.0 \\
\hline False-positive rate (\%) & 26.1 & 69.2 & 61.0 & 46.7 & 95.0 \\
\hline
\end{tabular}

${ }^{1}$ Ames, Ames test; MN, in vitro micronucleus assay; MLA, mouse lymphoma assay.

2 Sensitivity is defined as the percentage of known carcinogens that gave a positive result; when assessing a combination of more than one assay, a positive result in at least one assay is required.

${ }^{3}$ Specificity is defined as the percentage of known non-carcinogens that gave a negative result; when assessing a combination of more than one assay, a negative result in all tests is required. 
[1] K. Ji, T. Kogame, K. Choi, X. Wang, J. Lee, Y. Taniguchi, S. Takeda, A novel approach using DNA-repair-deficient chicken DT40 cell lines for screening and characterizing the genotoxicity of environmental contaminants, Environmental Health Perspectives 117 (2009) 1-9.

[2] J.-M. Buerstedde and S. Takeda, Increased ratio of targeted to random integration after transfection of chicken B cell lines, Cell 67 (1991) 179-188.

[3] M. Yamazoe, E. Sonoda, H. Hochegger, S. Takeda, Reverse genetic studies of the DNA damage resonse in the chicken B lymphocyte line DT40, DNA Repair (Amst) 3 (2004) 1175-1185.

[4] D.J. Kirkland, L. Henderson, D. Marzin, L. Müller, J.M. Parry, G. Speit, D.J. Tweats, G.M. Williams, Testing strategies in mutagenicity and genetic toxicology: an appraisal of the guidelines of the European Scientific Committee for Cosmetics and Non-Food Products for the evaluation of hair dyes, Mutation Research 588 (2005) 88-105.

[ 5 ] N. Baber, International conference on harmonisation of technical requirements for registration of pharmaceuticals for human use (ICH), Br. J. Clin. Pharmac 37 (1994) 401-404.

[6] D.J. Kirkland, M. Hayashi, D. Jacobson-Kram, P. Kasper, J.T. MacGregor, L. Müller, Y. Uno, Summary of major conclusions from the $4^{\text {th }}$ IWGT, San Francisco, 9-10 September, 2005, Mutation Research 627 (2007) 5-9.

[7] D.J. Kirkland, The role of the UKEMS in the development of testing guidelines, Mutagenesis 17 (2002) 451-455.

[8] D.J. Kirkland, M. Aardema, L. Henderson, L. Müller, Evaluation of the ability of a bettery of three in vitro genotoxicity tests to discriminate rodent carcinogens and non-carcinogens I. Sensitivity, specificity and relative predicitivity, Mutation Research 584 (2005) 1-256.

[9] J. Ashby, J.A. Styles, Does carcinogenic potency correlate with mutagenic potency in the Ames assay? Nature 271 (1978) 452-455.

[10] B.N. Ames, E.G. Gurney, J.A. Miller, H. Bartsch, Carcinogens as frameshift mutagens: metabolites and derivatives of 2-acetylaminofluorene and other aromatic amine carcinogens, Proc Natl. Acad. Sci USA 69 (1972) 3128-3132.

[11] B.N. Ames, W.E. Durston, E. Yamasaki, F.D. Lee, Carcinogens are mutagens: a simple test system combining liver homogenates for activation and bacteria for detection, Proc. Natl. Acad. Sci. USA 70 (1973) 2281-2285.

[12] B.N. Ames, F.D. Lee, W.E. Durston, An improved bacterial test system for the detection and classification of mutagens and carcinogens, Proc. Natl. Acad. Sci. USA 70 (1973) 782-786. 
[13] C.W. Lawrence, Cellular roles of DNA polymerase zeta and Rev1 protein, DNA Repair (Amst) 1 (2002) 425-435.

[14] M.C. Poirier, L.J. Schild, The genotoxicity of tamoxifen: extent and consequences, Kona, Hawaii, January 23, 2003, Mutagenesis 18 (2003) 395-399

[15] A. Mizutani, T. Okada, S. Shibutani, E. Sonoda, H. Hochegger, C. Nishigori, Y. Miyachi, S. Takeda, M. Yamazoe, Extensive chromosomal breaks are induced by tamoxifen and estrogen in DNA repair-deficient cells, Cancer Research 64 (2004) 3144-3147.

[16] B.D. Harfe, S. Jinks-Robertson, DNA polymerase $\zeta$ introduces multiple mutations when bypassing spontaneous DNA damage in Saccharomyces cerevisiae, Molecular Cell 6 (2000) 1491-1499.

[17] E. Sonoda, T. Okada, G.Y. Zhao, S. Tateishi, K. Araki, M. Yamaizumi, T. Yagi, N.S. Verkaik, D.C. van Gent, M. Takata, S. Takeda, Multiple roles of Rev3, the catalytic subunit of pol $\zeta$ in maintaining genome stability in vertebrates, EMBO J. 22 (2003) 3188-3197.

[18] D.J. Kirkland, M. Aardema, N. Banduhm, P. Carmichael, R. Fautz, J.-R. Meunier, S. Pfuhler, In vitro approaches to develop weight of evidence (WoE) and mode of action (MoA) discussions with positive in vitro genotoxicity results, Mutagenesis 22 (2007) 161-175.

[19] J.-M. Buerstedde, S. Takeda (Eds), Reviews and protocols in DT40 research, Springer, New York, 2006.

[20] E.M. Welch et al. PTC124 targets genetic disorders caused by nonsense mutations, Nature 447 (2007) 87-91.

[21] D.S. Auld, N. Thorne, W.F. Maguire, J. Inglese, Mechanism of PTC124 activity in cell-based luciferase assays of nonsense codon suppression, Proc. Natl. Acad. Sci. USA 106 (2009) 3585-3590.

[22] F.I. Abdullaev, R. Rivera-Luna, A. Garcia-Carranca, F. Ayala-Fierro, J.J. Espinosa-Aguirre, Cytotoxic effect of three arsenic compounds in HeLa human tumor and bacterial cells. Mutat Res 493 (2001) 31-8.

[23] D. Kirkland et al, How to reduce false positive results when undertaking in vitro genotoxicity testing and thus avoid unnecessary follow-up animal tests: Report of an ECVAM Workshop, Mutation Research 628 (2007) 31-55.

[24] J. Inglese, D.S. Auld, A. Jadhav, R.L. Johnson, A. Simeonov, A. Yasgar, W. Zheng, C.P. Austin, Quantitative high-throughput screening: a titration-based approach that efficiently identifies biological activities in large chemical libraries, Proc. Natl. Acad. Sci. USA 103 (2006) 11473-11478.

[25] M. Xia, R. Huang, K.L. Witt, N. Southall, J. Fostel, M.-H. Cho, A. Jadhav, C.S. Smith, J. Inglese, C.J. Portier, R.R. Tice, C.P. Austin, Compound cytotoxicity 
profiling using quantitative high-throughput screening, Environmental Health Perspectives 116 (2008) 284-291.

[26] M. Kirsch-Volders, L. Gonzalez, P. Carmichael, D. Kirkland, Risk assessment of genotoxic mutagens with thresholds: a brief introduction, Mutation Research 678 (2009) 72-75.

[27] S.P.M. Crouch, R. Kozlowski, K.J. Slater, J. Fletcher, The use of ATP bioluminescence as a measure of cell proliferation and cytotoxicity, J. Immunological Methods 160 (1993) 81-88.

[28] H.E. Bryant, N. Schultz, H.D. Thomas, K.M. Parker, D. Flower, E. Lopez, S. Kyle, M. Meuth, N.J. Curtin, T. Helleday, Specific killing of BRCA2-deficient tumours with inhibitors of poly(ADP-ribose) polymerase, Proc. Natl. Acad. Sci. USA 434 (2005) 913-917.

[29] H. Farmer, N. McCabe, C.J. Lord, A.N.J. Tutt, D.A. Johnson, T. B. Richardson, M. Santarosa, K.J. Dillon, I. Hickson, C. Knights, N.M.B. Martin, S.P. Jackson, G.C.M. Smith, A. Ashworth, Targeting the DNA repair defect in BRCA mutant cells as a therapeutic strategy, Proc. Natl. Acad. Sci. USA 434 (2005) 917-921.

[30] S. Jacob, F. Praz, DNA mismatch repair defects: role in colorectal carcinogenesis, Biochimie 84 (2002) 27-47.

[31] S.A. Martin, N. McCabe, M. Mullarkey, R. Cummins, D.J. Burgess, Y.

Nakabeppu, S. Oka, E. Kay, C.J. Lord, A. Ashworth, DNA polymerases as potential therapeutic targets for cancers deficient in the DNA mismatch repair proteins MSH2 or MLH1, Cancer Cell 17 (2010) 235-248.

[32] Y. Kumamoto-Yonezawa, R. Sasaki, Y. Suzuki, Y. Matsui, T. Hada, K. Uryu, K. Sugimura, H Yoshida, Y. Mizushina, Enhancement of human cancer cell radiosensitivity by conjugated eicosapentaenoic acid - a mammalian DNA polymerase inhibitor, Int. J. Oncol. 36 (2010) 577-584.

[33] H. Wang, W. Wu, H.W. Wang, S. Wang, Y. Chen, X. Zhang, J. Yang, S. Zhao, H.F. Ding, D. Lu, Analysis of specialized DNA polymerases expression in human gliomas: association with prognostic significance, Neuro Oncol (2010) doi:10.1093/neuonc/nop074.

[34] C.G. Murphy, M.E. Moynahan, BRCA gene structure and function in tumour suppression: a repair-centric perspective, Cancer J. 16 (2010) 39-47.

[35] E.M. Parry, J.M. Parry, C. Corso, A. Doherty, F. Haddad, T.F. Hermine, G. Johnson, M. Kayani, E. Quick, T. Warr, J. Williamson, Detection and characterization of mechanisms of action of aneugenic chemicals, Mutagenesis 17 (2002) 509-521. 


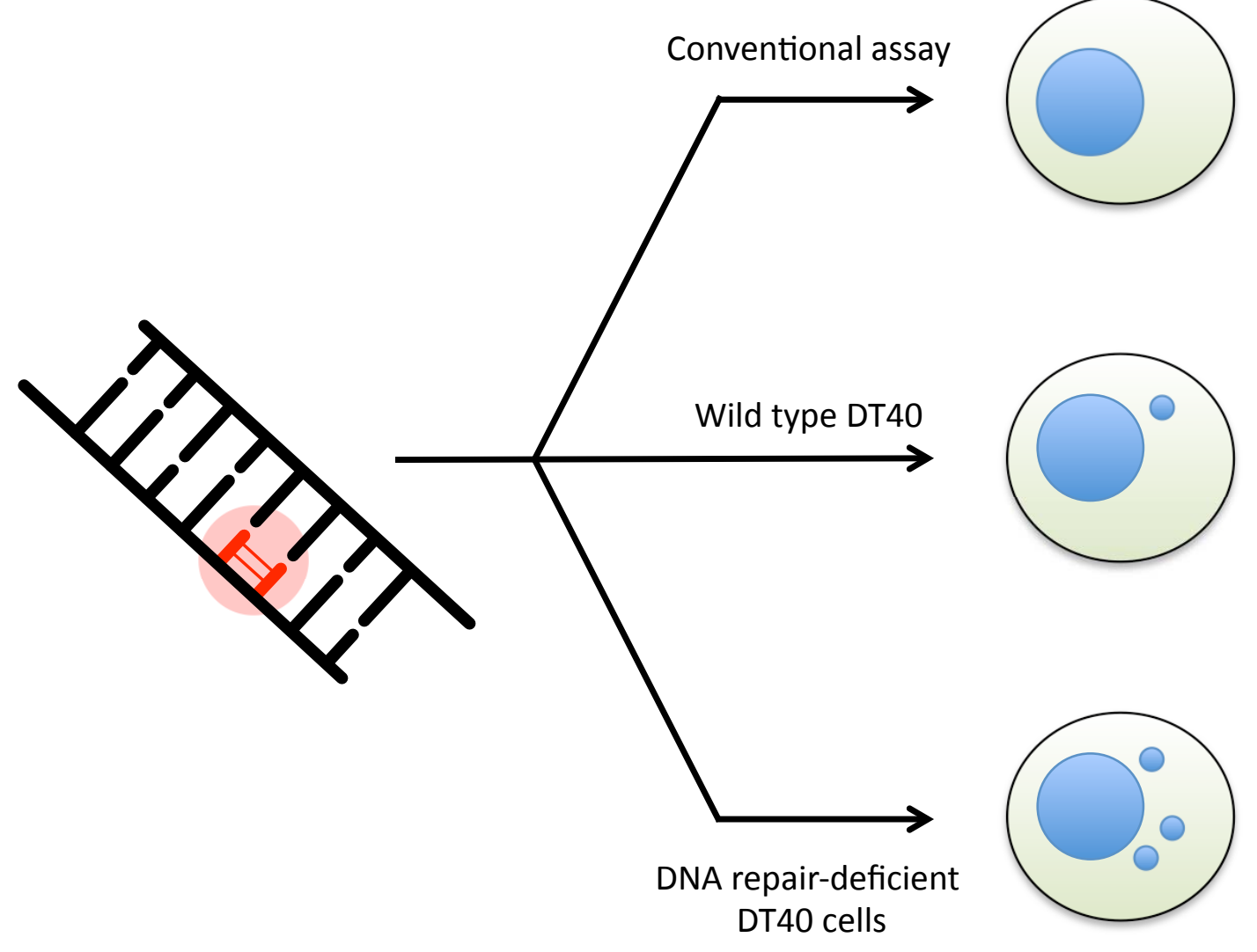

Figure 1. DT40 strains improve the sensitivity of the micronucleus assay. The conventional assay may fail to produce micronuclei due to $\mathrm{G} 1 / \mathrm{S}$ checkpoint activation following DNA damage, such as thymidine dimers induced by UV or a novel drug. DT40 lacks this checkpoint, and in DNA repair-deficient cells, unrepaired DNA damage results in increased numbers of micronuclei (small blue circles). 


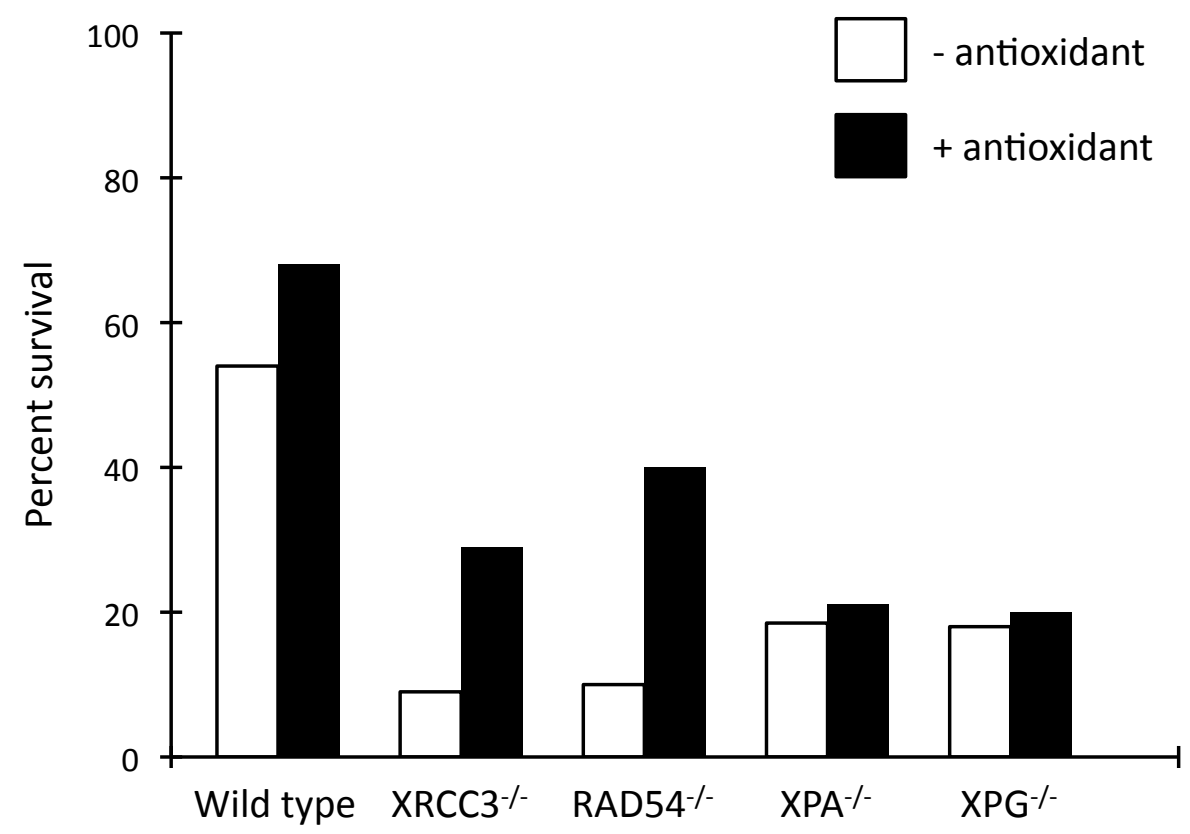

Figure 2. Survival of DT40 strains following arsenite exposure. Addition of an antioxidant to wild type cells reduces arsenite toxicity. This effect is mediated through antioxidant-dependent reversal of toxicity in homologous recombination-deficient strains (XRCC3 ${ }^{-/}$and RAD54 ${ }^{-/}$) but not in nucleotide excision repair-deficient strains (XPA ${ }^{-/}$and $\left.\mathrm{XPG}^{--}\right)$. This demonstrates that arsenite has two distinct mechanisms of genotoxicity. Adapted from [17]. 

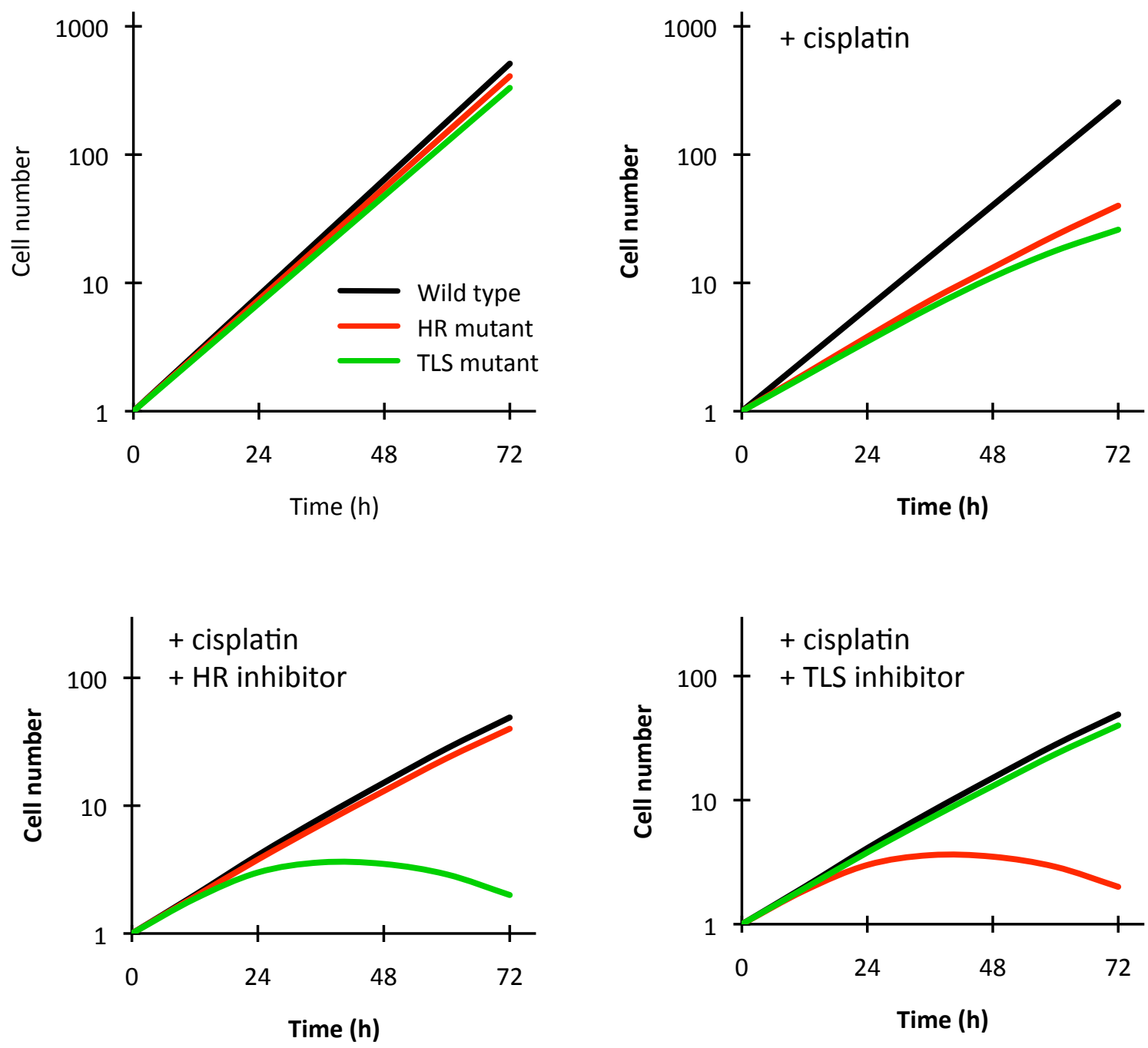

Figure 3. Simultaneous treatment of repair-deficient cells with a DNA damaging agent and an inhibitor of a redundant repair pathway causes a synergistic decrease in cellular viability. A: Wild type and mutant cells grow exponentially with a doubling time of $\sim 8 \mathrm{~h}$. B: Treatment with cisplatin results in reduced viability and a longer cell cycle. C, D: Since TLS and HR are largely redundant repair pathways, treatment of a TLS mutant with a HR inhibitor (and vice versa) is lethal. 